\title{
Unified NMPC for Multi-Variable Control in Smart Buildings
}

\author{
Mohamed Yacine Lamoudi* Mazen Alamir** \\ Patrick Béguery* \\ * Schneider-Electric Industries \\ Industries. 37, quai Paul Louis Merlin-38000 Grenoble-France. e-mail: \\ mohamed-yacine.lamoudi/patrick.beguery@fr.schneider-electric.com \\ Websites: http://www.schneider-electric.fr , \\ http://www.homesprogramme.com \\ ** CNRS-University of Grenoble, Gipsa-lab, BP 46, Domaine \\ Universitaire, 38400 Saint Martin d'Hères, France. Email: \\ Mazen.alamir@gipsa-lab.grenoble-inp.fr, \\ Web: http://www.mazenalamir.fr
}

\begin{abstract}
In this paper, the problem of minimizing energy consumption of a building zone under pre-assigned multi-variable comfort conditions and changing energy rate is addressed. The solution involves the use of a parameterized multi-variable Nonlinear Model Predictive Control (NMPC) that manages the actuation of heating/cooling, ventilation, lighting and blinds devices. Simulations of the resulting closed-loop in winter and summer seasons under varying rate profile are proposed to assess its efficiency. Moreover, a sensitivity analysis is conducted to show how the comfort level assignment impacts the level of energy consumption.
\end{abstract}

Keywords: Building Management System; NMPC, Consumption/comfort trade-off; Fixed-point iteration; Constrained control; Smart buildings.

\section{INTRODUCTION}

It is a fact that buildings energy consumption represents nearly $40 \%$ of total worldwide power consumption. This is the reason why, Building Energy Management Systems (BEMS) becomes a crucial paradigm in the context of rising energy price and climate warming. Although active research effort has been deployed these last years as it is attested by the excellent review by Dounis and Caraiscos [2009], many issues and challenges remain open.

A careful look on the available literature clearly shows the emergence of Model Predictive Control (MPC) as a particularly adapted choice since it enables to explicitly take into account, when computing the optimal scheduling of HVAC systems (Heating Ventilation and Air Conditioning), the available future predictions related to the weather, the energy rate variations and occupation forecast. The inclusion of these predictions in the scheduling algorithm is crucial given the thermal inertia of the buildings.

A hierarchical control structure is generally used in building control which can be split into two decision levels: building and zone levels. Building level is concerned with high level decisions. Zone level is concerned with regulation of environmental conditions in one zone of the building.

Although the idea of using MPC to address this problem is not new (Ferguson [1990] proposed a predictive controller for high inertia buildings where a stochastic approach has been used that led to remarkable results), the recent strategic emergence of this topics renewed the interest in this solution (Oldewurtel et al. [2010]). This is witnessed by the many recent works adopting such predictive solutions to address various related problems such as the control of micro-cogeneration system in domestic applications (Collazos et al. [2009]); temperature control (Gwerder and Tödtli [2009]), combined control of temperature and ventilation (Yuan and Perez [2006]). In Kolokotsa et al. [2009], an MPC controller has been designed that involves a BEMS system to regulate indoor temperature, dioxide carbon concentration and internal humidity ratio.

It goes without saying that an exhaustive overview of the related literature would be beyond the scope of this short communication. Instead, let us emphasize that the contribution of this work lies in the simultaneous handling of the following features:

- The multi-variable design that addresses the control of all the key quantities that influence the comfort level, namely, the temperature, the air quality $\left(\mathrm{CO}_{2}\right.$ level) and the lighting. The simultaneous handling of these variables increases considerably the size of the underlying problem making necessary to look carefully at the computational burden.

- The introduction of a simple and direct relationship between a pre-specified level of comfort and the corresponding minimal need for energy to meet it. Such a direct link will presumably be of tremendous 
importance in future coordination schemes as it is discussed later in the paper.

The paper is organized as follows. First, a brief recall on NMPC design is proposed in section 2. Section 3 briefly describes the structure of the dynamic model for a building zone. The comfort and actuator related operational constraints are described in section 4 while the cost function used in the NMPC design is described in section 5. Some implementation issues are discussed in section 7. Some validating simulation are proposed in section 8.2.2. By a direct use of the underlying scheme, a sensitivity analysis is finally proposed in section 9 in order the show the cost/comfort trade-off.

\section{RECALLS ON PREDICTIVE CONTROL}

In this section, the principle of NMPC is recalled for completeness. More detailed presentation can be found in the excellent paper by Mayne et al. [2000].

Consider a general dynamical systems governed by the following set of discrete-time equation:

$$
x(k+1)=f(x(k), u(k), w(k))
$$

where $(x, u, w) \in \mathbb{R}^{n} \times \mathbb{R}^{n_{u}} \times \mathbb{R}^{n_{w}}$ stand for the state, the control and the exogenous signal, respectively. MPC feedback control at instant $k$ is computed by first finding the optimal sequence of future open-loop controls $\tilde{u}^{o p t}(x(k)):=\left(u^{o p t^{T}}(k), \ldots, u^{o p t^{T}}(k+N-1)\right)^{T} \in \mathbb{R}^{N \cdot n_{u}}$ that minimizes some cost function $J(\tilde{u}, x(k))$ defined over the prediction horizon $[k, k+N]$ starting from the initial state $x(k)$, namely:

$\tilde{u}^{\text {opt }}(x(k))=\arg \min _{\tilde{u} \in \mathbb{R}^{N \cdot n_{u}}} J(\tilde{u}, x(k)) \quad$ under $\mathcal{C}(\tilde{u}, x(k)) \leq 0$ where $\mathcal{C}(\tilde{u}, x(k)) \leq 0$ gathers the set of operational constraints. The feedback at instant $k$ is then defined to be the first control $u^{o p t}(k)$ in the optimal sequence $\tilde{u}^{o p t}(k)$, namely:

$$
u(k)=K_{M P C}(x(k))=\Pi_{1}^{N} \cdot \tilde{u}^{o p t}(x(k))
$$

where $\Pi_{j}^{N} \in \mathbb{R}^{n_{u} \times\left(N \cdot n_{u}\right)}$ is the matrix that selects the $j$-th vector $u^{o p t}(k+j-1)$ in the sequence of $N$ concatenated vectors $\tilde{u}^{\text {opt }}(x(k))$. At the next sampling instant $k+1$, the new optimal sequence $\tilde{u}^{\text {opt }}(x(k+1))$ is computed and the first control $u^{o p t}(k+1)$ is applied during the sampling period $[k+1, k+2]$ and so on.

In the sequel, the dynamic model, the operational constraints and the cost function are successively described in order to completely characterize the control problem and its solution.

\section{DYNAMIC MODEL OF A ZONE}

In this section, we describe some key structural feature of the identified dynamic model of a building zone since they are needed to understand the fixed-point iteration used later in deriving the solution of the MPC related

\begin{tabular}{|c|c|c|c|}
\hline Variables & Description & unit & Output(s) \\
\hline$u_{h}$ & Heating ctrl & {$[-]$} & $T_{a i r}$ \\
\hline$u_{c}$ & Cooling ctrl & {$[-]$} & $T_{a i r}$ \\
\hline$u_{v}$ & ventilation control & {$[-]$} & $T_{\text {air }} / \mathrm{CO}_{2}$ \\
\hline$u_{l}$ & lighting control & {$[-]$} & $T_{a i r} / L$ \\
\hline$u_{b_{1}}$ & blind ctrl facade 1 & {$[-]$} & $T_{a i r} / L$ \\
\hline$u_{b_{2}}$ & blind ctrl facade 2 & {$[-]$} & $T_{\text {air }} / L$ \\
\hline$T^{e x t}$ & outdoor temperature & {$\left[{ }^{\circ} \mathrm{C}\right]$} & $T_{a i r}$ \\
\hline$\phi_{g l o b 1}$ & global irr. ${ }^{1}$ flux facade 1 & {$\left[\frac{W}{m^{2}}\right]$} & $T_{\text {air }} / L$ \\
\hline$\phi_{g l o b 1}$ & global irr. flux facade 2 & {$\left[\frac{W}{m^{2}}\right]$} & $T_{\text {air }} / L$ \\
\hline Occup & Number of occupants & {$[-]$} & $\mathrm{T}_{\text {air }} / \mathrm{CO}_{2}$ \\
\hline $\mathrm{CO}_{2}^{\text {ext }}$ & Outdoor $\mathrm{CO}_{2}$ level & {$[p p m]$} & $\mathrm{CO}_{2}$ \\
\hline$T_{a i r}$ & Indoor air temperature & {$\left[{ }^{\circ} \mathrm{C}\right]$} & - \\
\hline $\mathrm{CO}_{2}$ & Indoor $\mathrm{CO}_{2}$ level & {$[p p m]$} & - \\
\hline$L$ & Indoor illuminance & {$[L u x]$} & - \\
\hline
\end{tabular}
optimization problem. However, the complete details of
Table 1. Description of Input/Output and exogenous variables

the model are omitted since they result from classical arguments that can be found in existing references (Virk and Cheung [1995], Mustafaraja et al. [2010], Gwerder and Tödtli [2009], Kolokotsa et al. [2009]).

The identification of a structured model involving the key zone quantities (with two facades) leads to the following state space dynamic representation:

$$
\begin{aligned}
x^{+} & =A x+[B(\xi, w)] u+G w \\
y & =C x+[D(w)] u+F w \\
\xi & =H_{1} y+H_{2} w
\end{aligned}
$$

where $x$ is the state of the identified model, $y=$ $\left(T, C O_{2}, L\right) \in \mathbb{R}^{3}$ is the output vector gathering the physical key quantities, namely, temperature, $\mathrm{CO}_{2}$ level and light intensity $L . u \in \mathbb{R}^{6}$ regroups all the control input variables (see table 1). The vector: $w=$ $\left(T^{e x t}, \phi_{\text {glob1 } 1}, \phi_{\text {glob2 }}, \text { Occup }, C O_{2}^{e x t}\right)^{T}$ represents the exogenous variables (see table 1 ) where $\phi_{g l o b 1}$ and $\phi_{g l o b 2}$ characterize the global irradiance on the facades while Occup is the number of occupants in the zone. The term $[B(\xi, w)] u$ is a bilinear term $(B(\xi, w)$ is affine in its arguments) that describes the heat and $\mathrm{CO}_{2}$ transfer that depend not only on the actuator positions $u$ but also on the difference between indoor and external quantities $\left(T-T^{e x t}\right)$ and $\left(\mathrm{CO}_{2}-\mathrm{CO}_{2}^{e x t}\right)$ i.e: $\xi:=\left[T-T^{e x t}, \mathrm{CO}_{2}-\mathrm{CO}_{2}^{e x t}\right]^{T}$. This explain the form of $(5)$

In the sequel, the notation $X\left(\tilde{u}, \tilde{w}, x_{0}\right)\left[\operatorname{resp} . Y\left(\tilde{u}, \tilde{w}, x_{0}\right)\right]$ is used to denote the trajectory of the state [resp. output] for a given sequences $\tilde{u}$ and $\tilde{w}$ of future evolutions of $u$ and $w$ respectively and starting from the initial state $x_{0}$.

\section{OPERATIONAL CONSTRAINT}

In accordance with existing standard (EN1 [2005]), the comfort level is linked to an admissible set to which the output $y$ has to belong. This admissible set obviously depends on the occupation indicator, the current season and the nature of the zone under consideration. These consideration leads to the following constraints:

$$
y(k) \in\left[\underline{y}(k)-\frac{1-c}{2} \Delta_{y}, \bar{y}(k)+\frac{1-c}{2} \Delta_{y}\right]
$$




\begin{tabular}{|c|c|c|c|c|c|c|}
\hline & \multicolumn{2}{|c|}{$T_{\text {air }}$} & \multicolumn{2}{c|}{$\mathrm{CO}_{2}$} & \multicolumn{2}{c|}{ Lum } \\
\hline & $\min$ & $\max$ & $\min$ & $\max$ & $\min$ & $\max$ \\
\hline Occupation period & 19 & 22 & - & 900 & 500 & - \\
\hline Vacant period & 5 & 30 & - & - & - & - \\
\hline
\end{tabular}

Table 2. Nominal comfort region

where $y(k) \in \mathbb{R}^{3}$ and $\bar{y}(k) \in \mathbb{R}^{3}$ are the nominal lower and upper bounds that implicitly depends on the occupation indicator (through the time argument $k$ ) [see (Morosan et al. [2010], Collazos et al. [2009]) for examples of such bounds] while $c \in[0,1]$ denote the comfort level. Note that if $c=1$, the nominal bounds are used while the use of $c=0$ enlarges the admissible domain by decreasing the lower bound and increasing the upper bound by the same amount $\Delta_{y} \in \mathbb{R}^{3}$. Note that this corresponds to a less stringent requirements on the fulfillment of the nominal requirements. Table 2 shows typical values of $y$ and $\bar{y}$ depending on the occupation indicator.

Saturation constraints on the actuators are also considered using standard constraints of the form:

$$
\left[A_{\text {sat }}\right] u(k) \leq b_{\text {sat }}
$$

which may include both saturation the value of $u$ as well as on its rate of change. Here, normalized vector are considered leading to $u(k) \in[0,1]^{6}$ for all $k$.

\section{COST FUNCTION}

In this section, the cost function $J(\cdot, x(k))$ that is used at each instant $k$ to compute the optimal sequence as recalled in section 2 is described. This cost function is defined based on the following given knowledge:

- The prediction of the external exogenous signal

- The current state $x(k)$ of the system model (obtained via classical dynamic observer.

- The normalized characteristic power consumption $\alpha_{j}$ of each actuator $j\left(\alpha_{j} u_{j}\right.$ represents the power)

- The prediction of the normalized energy rate profile $\tilde{\beta}_{j}$ for each actuator type $j$.

- The comfort related bounds profiles $\underline{y}(k+i)$ and $\bar{y}(k+$ i) for $i \in\{1, \ldots, N\}$

based on the above notation, two cost functions can be defined over the prediction horizon $[k, k+N]$ :

- The total energy consumption corresponding to some given control profile $\tilde{u}$ :

$$
J_{1}(\tilde{u}, x(k)):=\sum_{i=0}^{N-1} \sum_{j=1}^{n_{u}} \alpha_{j} u_{j}(k+i)
$$

- The corresponding total energy invoice:

$$
J_{2}(\tilde{u}, x(k)):=\sum_{i=0}^{N-1} \sum_{j=1}^{n_{u}} \beta_{j}(k+i)\left[\alpha_{j} u_{j}(k+i)\right]
$$

Note that in both case, the cost function, et instant $k$, is affine in the control sequence and takes the form:

$$
J_{i}(\tilde{u}, x(k))=L(k)^{T} \tilde{u} \quad ; \quad L(k) \in \mathbb{R}^{1 \times N \cdot n_{u}}
$$

and the NMPC-related optimization problem at instant $k$ becomes:

$$
\begin{aligned}
& \min _{\tilde{u}} L(k)^{T} \tilde{u} \quad \text { s.t. } \forall i \in\{1, \ldots, N\} \\
& Y(k+i, \tilde{u}, \tilde{w}, x(k)) \leq \bar{y}(k+i)+\frac{1-c}{2} \Delta_{y} \\
& Y(k+i, \tilde{u}, \tilde{w}, x(k)) \geq \underline{y}(k+1)-\frac{1-c}{2} \Delta_{y} \\
& {\left[A_{\text {sat }}\right] u(k+i) \leq b_{\text {sat }}}
\end{aligned}
$$

Unfortunately, this optimization problem is not necessarily feasible and may lead to untractable situation under certain conditions. That is the reason why the following relaxed optimization problem is formulated in which two dynamic slack variable profiles $\delta^{+}(\cdot) \geq 0$ and $\delta^{-}(\cdot) \geq 0$ are introduced to soften the constrained when appropriate:

$$
\begin{aligned}
& \min _{\tilde{u}} L(k)^{T} \tilde{u}+\rho \cdot\left(\tilde{\delta}^{+}+\tilde{\delta}^{-}\right) \quad \text { s.t. } \forall i \in\{1, \ldots, N\}(15) \\
& Y(k+i, \tilde{u}, \tilde{w}, x(k)) \leq \bar{y}(k+i)+\frac{1-c}{2} \Delta_{y}+\delta^{+} \\
& Y(k+i, \tilde{u}, \tilde{w}, x(k)) \geq \underline{y}(k+1)-\frac{1-c}{2} \Delta_{y}-\delta^{-} \\
& {\left[A_{\text {sat }}\right] u(k+i) \leq b_{\text {sat }}} \\
& \delta^{+}(k+i) \geq 0 \quad, \quad \delta^{-}(k+i) \geq 0
\end{aligned}
$$

note that these slack variable are heavily weighted through $\rho>0$ in order to avoid unnecessary constraint violation. This new problem is obviously always feasible since $\delta^{+}$ and $\delta^{-}$can always be taken sufficiently large to satisfy the constraints (16)-(17). Note also that the new formulation involves the vector of unknowns $\tilde{z}$ give by:

$$
\tilde{z}=\left(\tilde{u}^{T}, \tilde{\delta}^{+}, \tilde{\delta}^{-}\right) \in \mathbb{R}^{N n_{u}} \times \mathbb{R}^{3 N} \times \mathbb{R}^{3 N}
$$

It is worth underlying that the above optimization problem is not necessarily convex since the terms $Y(k+i, \tilde{u}, \tilde{w})$ are not affine in $\tilde{u}$ because of the bilinear terms $[B(\xi, w)] u$ appearing in (3). Moreover, this problem has to be solved quite rapidly since a new problem is defined at each sampling period. Therefore, a classical incremental gradient based smooth iteration would be unsuccessful here. That is the reason why, in the next section, an efficient fixed point iteration is derived to obtain a fast solution of the above problem.

\section{SOLVING THE OPTIMIZATION PROBLEM}

As mentioned at the end of the preceding section, it is the bilinear term $[B(\xi, w)] u$ appearing in (3) that prevents (15)-(19) from being a Linear Programming (LP) problem. The idea is then to define for any given $\xi$-profile $\tilde{\xi}^{*}$, the following LP problem, denoted hereafter by $L P\left(\tilde{\xi}^{*}\right)$ :

$$
\begin{aligned}
& \min _{\tilde{u}} L(k)^{T} \tilde{u}+\rho \cdot\left(\tilde{\delta}^{+}+\tilde{\delta}^{-}\right) \quad \text { s.t. } \forall i \in\{1, \ldots, N\}(21) \\
& Y^{\left(\tilde{\xi}^{*}\right)}(k+i, \tilde{u}, \tilde{w}, x(k)) \leq \bar{y}(k+i)+\frac{1-c}{2} \Delta_{y}+\delta^{+}(22 \\
& Y^{\left(\tilde{\xi}^{*}\right)}(k+i, \tilde{u}, \tilde{w}, x(k)) \geq \underline{y}(k+1)-\frac{1-c}{2} \Delta_{y}-\delta^{-}(23) \\
& {\left[A_{\text {sat }}\right] u(k+i) \leq b_{\text {sat }}} \\
& \delta^{+}(k+i) \geq 0 \quad, \quad \delta^{-}(k+i) \geq 0
\end{aligned}
$$

where $Y^{\left(\tilde{\xi}^{*}\right)}(k+i, \tilde{u}, \tilde{w}, x(k))$ is the output trajectory of the following fictitious system: 


$$
\begin{aligned}
x^{+} & =A x+\left[B\left(\xi^{*}, w\right)\right] u+G w \\
y & =C x+[D(w)] u+F w
\end{aligned}
$$

in which $\xi^{*}(\cdot)$ is no more linked to $x$ and $w$ but is a priori given.

The new problem $L P\left(\tilde{\xi}^{*}\right)$ is a linear programming problem since the terms $Y^{\left(\tilde{\xi}^{*}\right)}(k+i, \tilde{u}, \tilde{w}, x(k))$ are now affine in $\tilde{u}$. Therefore, for each candidate profile, $\tilde{\xi}^{*}, L P\left(\tilde{\xi}^{*}\right)$ can be efficiently solved using standard LP solvers to obtain the corresponding control profile $\tilde{u}^{*}\left(\tilde{\xi}^{*}\right)$ solution of $L P\left(\tilde{\xi}^{*}\right)$. Injecting this control profile in the original model (3)-(5) leads to a corresponding $\xi$-profile, denoted by:

$$
\tilde{\xi}^{\sharp}=\mathcal{S}\left(\tilde{\xi}^{*}\right)
$$

which, according to (5) is given by:

$$
\xi^{\sharp}(k+i)=H_{1} Y\left(k+i, \tilde{u}^{*}\left(\tilde{\xi}^{*}\right), \tilde{w}, x(k)\right)+H_{2} w(k+i)
$$

It goes without saying that for arbitrarily chosen profile $\tilde{\xi}^{*}$, the real-system profile $\tilde{\xi}^{\sharp}$ would be obviously different from $\tilde{\xi}^{*}$. However, if one can find $\tilde{\xi}^{*}$ such that the following fixed point equality holds:

$$
\tilde{\xi}^{*}=\mathcal{S}\left(\tilde{\xi}^{*}\right)
$$

then the optimal solution $\tilde{u}^{*}\left(\tilde{\xi}^{*}\right)$ is obviously solution of the original problem (15)-(19). We clearly have to solve the fixed point problem (29).

In order to do this, we suggest the following intuitive iteration starting from some arbitrary profile $\tilde{\xi}^{(0)}$ :

$$
\tilde{\xi}^{(i+1)}=\mathcal{S}\left(\tilde{\xi}^{(i)}\right)
$$

with the following stopping condition:

$$
\begin{aligned}
& \left\|\tilde{u}^{*}\left(\tilde{\xi}^{(i)}\right)-\tilde{u}^{*}\left(\tilde{\xi}^{(i-1)}\right)\right\| \leq \eta_{u} \quad \text { and } \\
& \left\|\tilde{Y}\left(\tilde{u}^{*}\left(\tilde{\xi}^{(i)}\right), \tilde{w}, x(k)\right)-\tilde{Y}\left(\tilde{u}^{*}\left(\tilde{\xi}^{(i-1)}\right), \tilde{w}, x(k)\right)\right\| \leq \eta_{y}
\end{aligned}
$$

where $\eta_{u}>0$ and $\eta_{y}>0$ are small thresholds. The stopping condition (31) simply means that one reach an approximate solution $\tilde{\xi}^{(i)}$ of the fixed point equation (29) which means that $\tilde{u}^{*}\left(\tilde{\xi}^{(i)}\right)$ is an approximation of the optimal solution of the original problem that may be rendered as close as desired to optimality by taking smaller thresholds $\eta_{u}$ and $\eta_{y}$. The convergence of this fixed point iteration is investigated in section 8.1.

\section{SOME IMPLEMENTATION ISSUES}

It is known that the number of inequality constraints has a crucial impact on the computational burden of LP problems. For instance, if one considers the case of minimizing energy cost over a prediction horizon length of $N=720$ (a 24 hours horizon with a sampling time of 1 min.), the number of decision variables is given by $N_{d v}=\left(n_{u}+\right.$ $\left.2 n_{y}\right) \cdot N=8640$ [see (20)] and the number of operational constraints is given by $N_{c}=\left(2 n_{y}+4 n_{u}\right) \cdot N=21600$. It comes out clearly that one is faced with a high dimensional problem that may be difficult (if not impossible to solve in the allocated time).

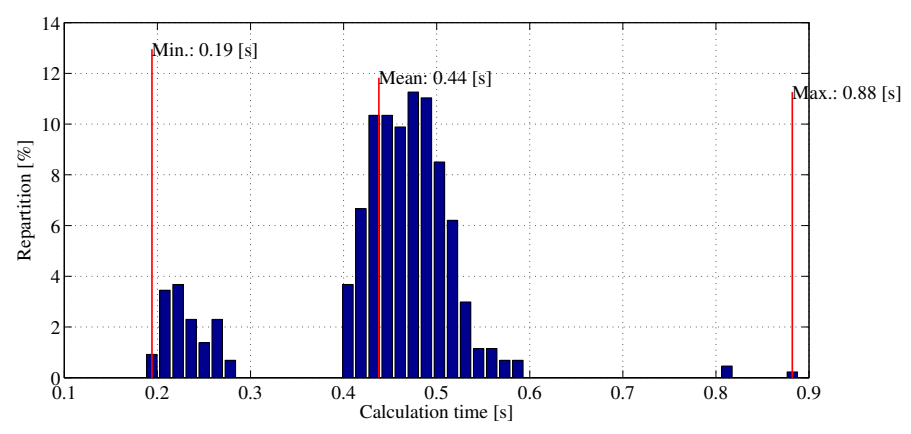

Fig. 1. Computation time repartition for $N=720, T_{u}=$ $10, T_{y}=10, \eta_{u}=10^{-2}, \eta_{y}=10^{-1}$. This computation time enables a control updating period of $2 \mathrm{sec}$

In order to reduce the number of inequality constraints, one may use (potentially) different under-sampling rates for control updating and for output constraints checking. More clearly, one may use open-loop time profile that maintains the control input constant during $T_{u}$ and an output constraints checking sampling period of $T_{y}$. Note that this does not mean that the control updating period is equal to $T_{u}$. The latter may be as small as desired provided that it remains greater than the computation time (see below). By doing so, the problem size drastically decreases. For instance, with $T_{u}=10 \mathrm{~min}$. and $T_{y}=10 \mathrm{~min}$., the dimension of the decision variable drops to $N_{d v}=864$ while the number of operational constraints becomes $N_{c}=2160$.

As a matter of fact, lower bound on $\mathrm{CO}_{2}$ and upper bound on $L$ doesn't exist and no bounds on these outputs prevail during vacant periods (refer to table 2). Therefore, their associated constraints and related relaxation variables disappear.

Figure 1 shows the computation time obtained for $N=$ $720, T_{u}=10$ and $T_{y}=10$, the maximum compute time $(0.88 \mathrm{sec})$ corresponds to the first iteration.. The next ones are much smaller because of the warm conditioning. This enables a control updating period of $2 \mathrm{sec}$ for instance.

These results are obtained on an Intel ${ }^{\circledR}$ Pentium ${ }^{\circledR} \mathrm{M}$ processor $1.86 \mathrm{GHz}, 0.99$ Go RAM, we used ILOG CPLEX 12.1.

\section{SIMULATION RESULTS}

\subsection{Algorithm convergence}

Although there is no formal proof of the convergence of the fixed-point iteration 30, Figure 2 shows the evolution of $\epsilon_{u}=\left\|\tilde{u}^{(i)}-\tilde{u}^{(i-1)}\right\|$ and $\epsilon_{y}=\left\|\tilde{y}^{(i)}-\tilde{y}^{(i-1)}\right\|$ when the iterations start from 100 different random initial guesses $\tilde{\xi}^{(0)}$. The algorithm converges even when one starts with quite unrealistic initial guesses. It can be noticed that the algorithm converges to optimal solution very quickly when starting form a realistic solution. With a warm start(i.e: initializing the algorithm a time $k$ with the optimal solution found at $k-1$ ) convergence is generally achieved in one or two iterations depending on the tolerances $\eta_{u}$ and $\eta_{y}$. Moreover $\eta_{u}$ and $\epsilon_{y}$ are always monotonously decreasing. 


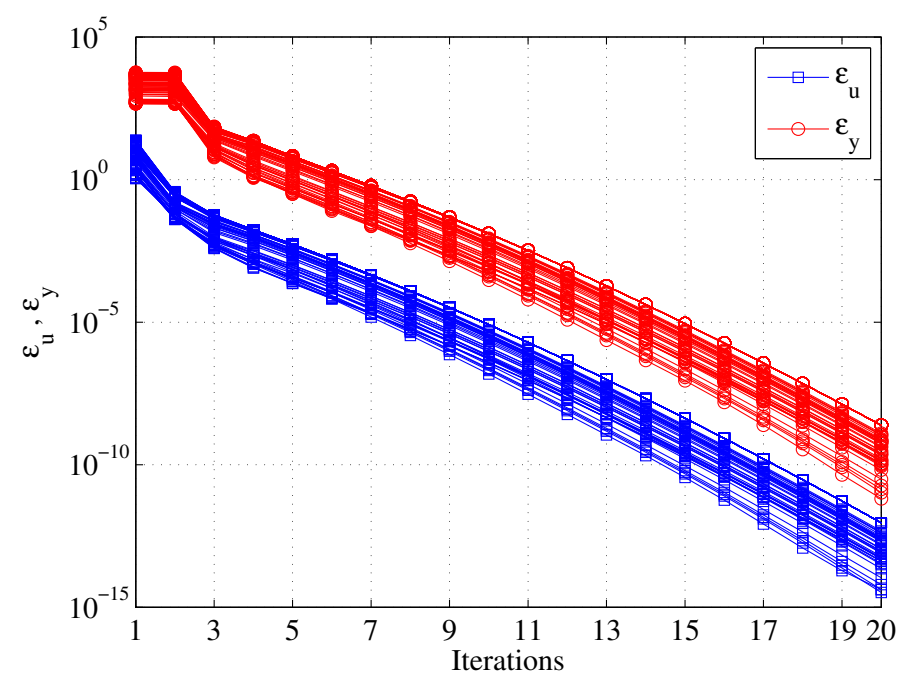

Fig. 2. Algorithm convergence: Evolution of $\epsilon_{u}$ and $\epsilon_{y}$ when starting from 100 random initial guesses $\tilde{\xi}^{0}$.

\subsection{Simulation conditions}

NMPC closed-loop simulations have been performed using a prediction horizon of $N=720 \mathrm{~min}$ (12 hours), with control and constraint checking parameters of $T_{u}=$ $T_{y}=10 \mathrm{~min}$ (see section 7) with an updating control period of $10 \mathrm{~min}$. The results are obtained assuming all predictions including those on meteorological conditions and occupation profile are perfectly known in advance. In the varying rate case, the energy cost is 2 times higher between $06 \mathrm{~h} 00$ and $22 \mathrm{~h} 00$ (this corresponds to the French tarification full hours/empty hours ${ }^{2}$ ). The simulations are performed using a weather data provided by SIMBADdatabase for the city of Paris ${ }^{3}$, the days chosen are $1^{s t}$ January for winter simulations and $1^{\text {st }}$ July for summer simulations.

Winter Simulations Figure 3 shows the anticipative effect of the controller which enables the optimal heating start time to be found (here 06h10) in the constant rate case. In the varying rate case, the behavior is quite different since temperature is increasing before $06 \mathrm{~h} 00$, when energy is cheaper. Note that in this case, the optimal behavior is to start heating at $00 \mathrm{~h} 50 \mathrm{~m}$ and stop heating at $06 \mathrm{~h} 00$ (that is, almost 5 hours before the heating start in the constant rate case). The temperature then decreases to join the temperature comfort region in occupation hours (less than $28^{\circ} \mathrm{C}$ ). Note also that the ventilation is used at the minimum necessary level to ensure a good air quality level. This is in order to introduce as less as possible of new fresh air.

Summer Simulations Summer simulations show also good results, since the anticipative effect of the NMPC is clearly visible. Remark in this case that the ventilation control is activated during night in order to introduce fresh air to "pre-cool" the zone which is made possible by the anticipation capability that is inherent to the use of Model Predictive Control. Moreover the night cooling is activated more longer in the variable rate case. Note

2 in French: heures pleines / heures creuses

3 weather recorded in 1998
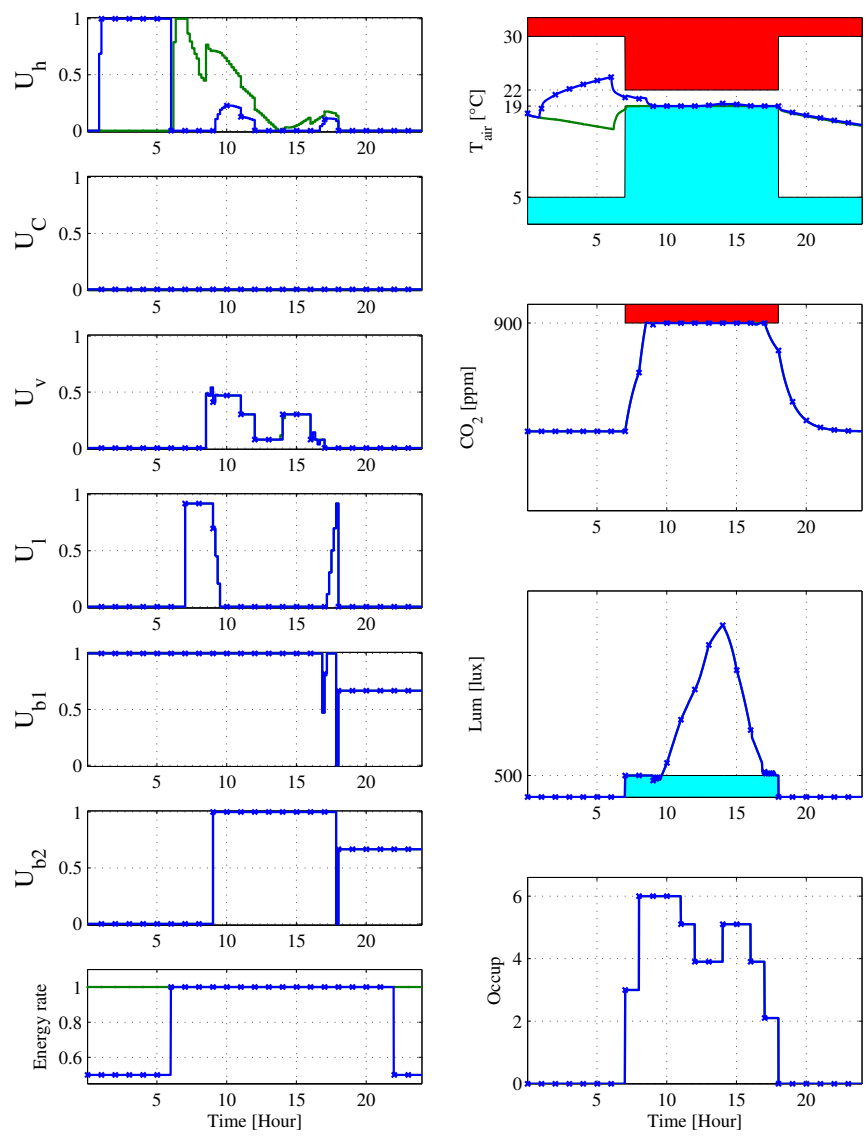

$\mathrm{C}^{\text {st }}$ Energy rate $\longrightarrow$ Variable Energy rate

Fig. 3. Closed-loop behavior in winter scenario for constant and variable (cross) energy rate cases

that this underline the explicit handling of the coupling effects between the different variables and actuators (here ventilation and cooling control) The blinds are open just to get enough light in the zone and ensure minimum heat introduction via windows.

\section{ENERGY CONSUMPTION VS. COMFORT: A TRADEOFF}

At this point, a predictive controller has been designed. We remind that the controller takes into account weather and occupancy forecast as well as energy rate to find the best control sequence. Considering that this operation can be performed for different comfort indexes $c \in[0,1]$ and denoting the optimal total energy cost on the horizon $N$ for a certain comfort level by Con(c), one can obtain the function that represents the optimal total energy cost for each $c$. The allure of this function is depicted in Figure 5 in winter conditions. Here $N=1440 \mathrm{~min}$ (24 hours) and $\Delta_{y}=(20,1000,1300)$. The power consumption of equipments are given by $\alpha=(3.5,2,0.15,0,0)[k W h]$. This information can be crucial when looking for a Consumption/comfort trade-off in a constrained context.

\section{CONCLUSION AND FURTHER WORKS}

In this paper, an NMPC controller has been proposed to control both HVAC, lighting and blinds systems in an 

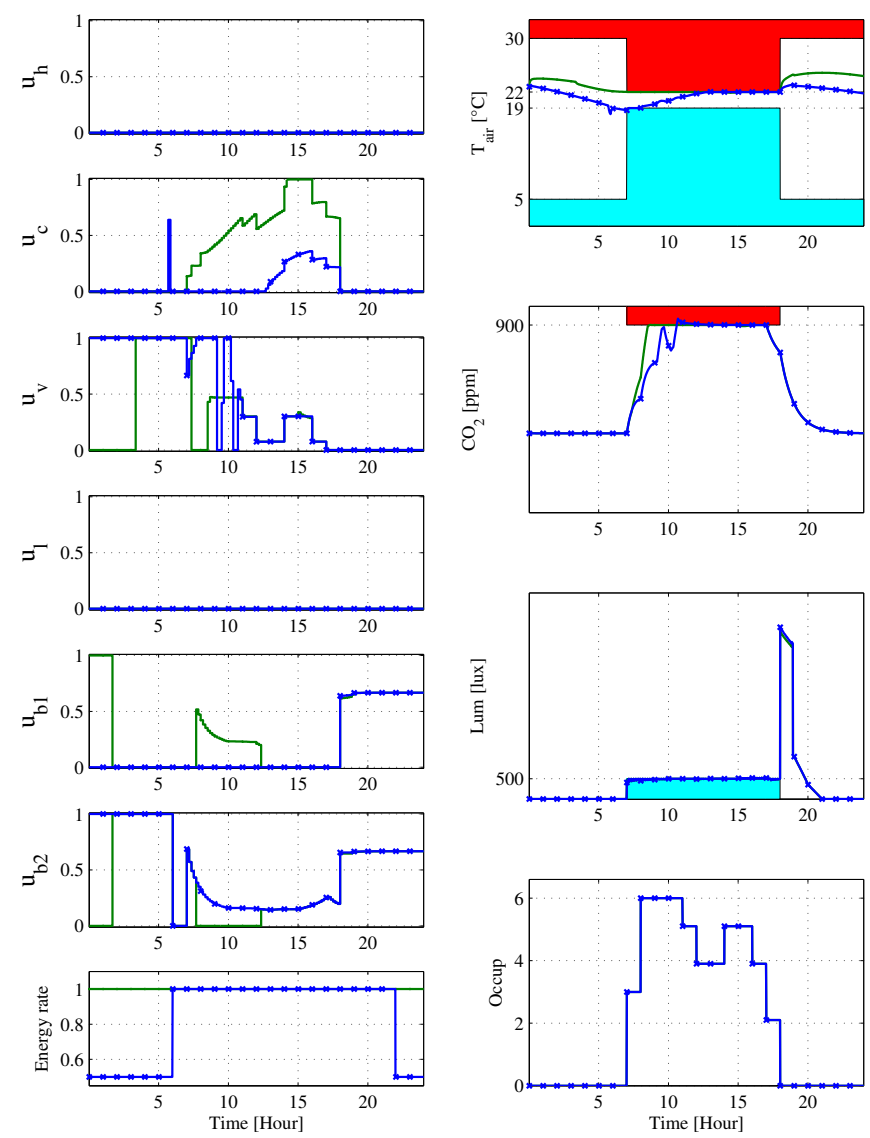

$\longrightarrow \mathrm{C}^{\text {st }}$ Energy rate $\longrightarrow$ Variable Energy rate

Fig. 4. Closed-loop behavior in summer scenario for constant and variable (cross) energy rate cases

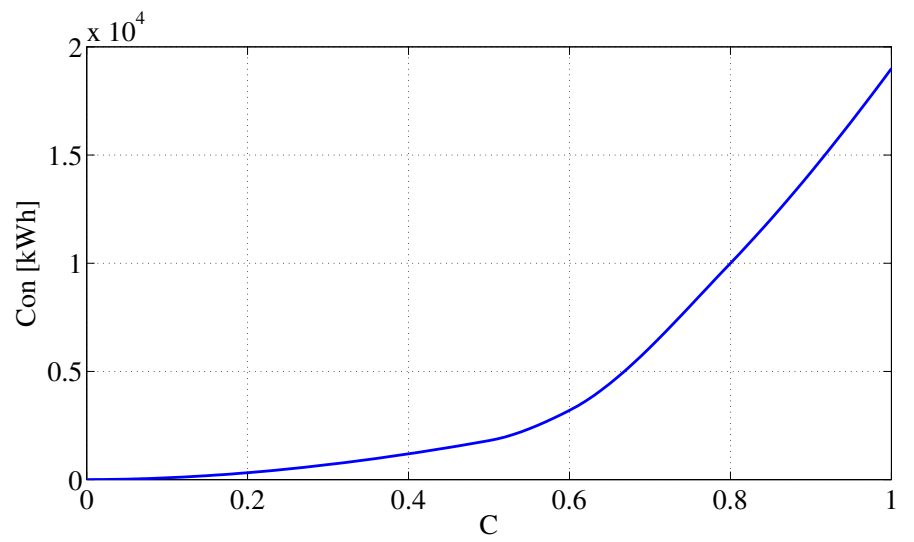

Fig. 5. Energy consumption vs. comfort index

efficient and coordinated manner ensuring optimal energy consumption/invoice while meeting comfort requirements. Efficient and simple solution to the underlying optimization problem has been proposed through an LP based fixed-point iteration. The results obtained by simulations in winter and summer seasons with a constant or variable rate context proves the benefits of the approach. The comfort level was described via a unique aggregated indicator that results from a simple parametrization of the comfort region domain, this parametrization can be improved in further works considering for example non symmetric and/or non homogeneous comfort degradation on $T_{a i r}$, $\mathrm{CO}_{2}$ and $L$ without any change in the remaining solution. Further studies will focuss on extending the control to a whole building, to this end, the use of the cost/comfort map $\operatorname{Con}(\cdot)$ 's emanating from the zones can be of great interest since they provide the building level with a precious and condensed information about the zones situation. This may reduce the complexity of the decision making at the high level stages.

\section{Acknowledgement}

This work is a part of the French collaborative program HOMES (http://www.homesprogramme.com). HOMES program is funded by OSESO (http://www.oseo.fr).

\section{REFERENCES}

Standard: En15251 -indoor environmental input parameters for design and assessment of energy performance of buildings addressing indoor air quality, thermal environment, lighting and acoustics, August 2005.

A. Collazos, F. Maréchal, and C. Gähler. Predictive optimal management method for the control of polygeneration systems. Computers $\& 5$ Chemical Engineering, 33: 1584-1592, May 2009.

A.I. Dounis and C. Caraiscos. Advanced control systems engineering for energy and comfort management in a building environment-a review. Energy Conversion \& Management, 13:1246-1261, August-September 2009.

A. M. N. Ferguson. Predictive Thermal Control Of Building Systems. PhD thesis, Ecole Polytechnique Federale de Lausanne, 1990.

M. Gwerder and J. Tödtli. Patent, system for controlling room temperature in building using predictive control, April 2009.

D. Kolokotsa, A. Pouliezos, G. Stavrakakis, and C. Lazos. Predictive control techniques for energy and indoor environmental quality management in buildings. Building and Environment, 44:1850-1863, September 2009.

D. Q. Mayne, J. B. Rawlings, C. V. Rao, and P. O. M. Scokaert. Constrained model predictive control: Stability and optimality. Automatica, 36:789-814, 2000.

P.-D. Morosan, R. Bourdais, D. Dumur, and J. Buisson. Building temperature regulation using a distributed model predictive control. Energy \& Buildings, 42:1445 - 1452, 2010. ISSN 0378-7788.

G. Mustafaraja, J. Chena, and G. Lowry. Development of room temperature and relative humidity linear parametric models for an open office using bms data. Energy E Buildings, 42:348-356, March 2010.

F. Oldewurtel, D. Gyalistras, M. Gwerder, C. N. Jones, A. Parisio, V. Stauch, B. Lehmann, and M. Morari. Increasing energy efficiency in building climate control using weather forecasts and model predictive control. In 10th REHVA World Congress Clima, Antalya, Turkey, May 2010.

G.S. Virk and J.Y.M. Cheung. Practical stochastic multivariate identification for buildings. Appl. Math. Modeling, 19:622-636, October 1995.

S. Yuan and R. Perez. Multiple-zone ventilation and temperature control of a single-duct hvav system using model predictive strategy. Energy \& Buildings, 38:12481261, 2006. 